\title{
Gigantomastia gestacional: relato de caso e revisão bibliográfica
} Gestational gigantomastia: case report and bibliographic review Gigantomastia de la gestación: relato de un caso y revisión de la literatura

Mário Rogério da Silva Santos ${ }^{1}$, Alessandra Santos dos Reis², José Aroldo Lima Gonçalves-Filho ${ }^{*}$

Palavras-chave: Complicações na Gravidez Mamoplastia Doenças Mamárias Bromocriptina

Keywords: Pregnancy Complications Mammoplasty Breast Diseases Bromocriptine

Palabras clave: Complicaciones del Embarazo Mamoplastia Enfermedades de la Mama Bromocriptina

\section{Resumo}

A gigantomastia é uma condição clínica rara, pouco descrita na literatura. Apesar da etiologia desconhecida, tem sido associada às respostas aos hormônios gestacionais, com maior incidência em mulheres multíparas e caucasianas. Apresenta altas taxas de recorrência em gestações futuras. 0 quadro frequentemente está associado à dor incapacitante, a alterações posturais, a úlceras e à necrose cutânea, favorecendo 0 aparecimento de infecções. Os autores relatam um caso clínico ocorrido em quinta gestação, expondo a conduta da Equipe de Saúde da Família durante 0 acompanhamento pré-natal e a inibição da lactação com bromocriptina imediatamente após o parto e o preparo para mamoplastia redutora em segundo tempo.

\section{Abstract}

Gigantomastia is a rare clinical condition, with few cases reported in the literature. Although it has an unknown etiology, it has been associated with responses to pregnancy hormones, most frequently in multiparous and Caucasian women. It presents great capacity to recur in future pregnancies. This condition is often associated with disabling pain, postural disorders, ulcers and skin necrosis, leading to breast infections. The authors report one case occurred at the fifth pregnancy, explaining management of the Family Health Team during the prenatal and inhibition of lactation with bromocriptine immediately after childbirth, and breast reduction in preparation for the second time.

\section{Resumen}

La gigantomastia es una rara condición clínica, raramente descrita en la literatura. Aunque de etiología incierta, se ha sido asociada con las respuestas a las hormonas del embarazo, ocurre con mayor frecuencia en mujeres multíparas y las caucásicas. Presenta altas tasas de recurrencia en futuros embarazos. Se asocia a menudo con dolor incapacitante, trastornos posturales, úlceras y necrosis de la piel, favoreciendo la aparición de infecciones. Los autores presentan un caso ocurrido en quinto embarazo, exponiendo la conducta del Equipo de Salud de la Familia durante el prenatal y la inhibición de la lactancia con bromocriptina inmediatamente después del parto y la preparación para el manejo quirúrgico.
'Escola Nacional de Saúde Pública da Fundação Oswaldo Cruz (ENSP/FIOCRUZ).mariorssantos@uol.com.br Escola Nacional de Saúde Pública da Fundação Oswaldo Cruz (ENSP/FIOCRUZ). alessandra291274@yahoo.com.br ${ }^{3}$ Polícia Militar do Estado do Rio de Janeiro. goncalvesfilho@nutmed.com.br

*Autor correspondente.

Fonte de financiamento: nenhuma.

Conflito de interesses: declararam não haver.

Recebido em: 20/06/2011

Aprovado em: 18/09/2011 


\section{Introdução}

A gigantomastia é uma condição clínica de etiologia incerta, caracterizada pelo aumento difuso, maciço e incapacitante das mamas, propiciando necrose local, infecção, úlceras e sangramentos, além do risco aumentado de morbimortalidade ${ }^{1}$.

Tal enfermidade ocorre em 1/100.000 gestações, embora tenha sido observada por outros autores em proporções menores, como 1/28.000 gestações ${ }^{2,3}$. O crescimento exuberante e rápido, seja uni ou bilateralmente, é mais comum entre gestantes adolescentes e parece estar relacionado a uma resposta exacerbada dos receptores mamários aos hormônios gestacionais, os quais se encontram com níveis séricos normais ${ }^{4,5}$.

Os principais fatores de risco associados à gigantomastia não têm sido bem identificados, embora acometa com maior frequência multíparas e caucasianas ${ }^{3}$. $\mathrm{O}$ crescimento patológico das mamas e sua progressão variam de caso a caso. O tratamento baseia-se no conforto da paciente, no acompanhamento pré-natal e no uso de supressor de prolactina (bromocriptina) após o parto. Tem sido reportado aborto espontâneo em primeiro trimestre gestacional e, em alguns casos, apresentação da doença apenas no segundo ou terceiro trimestres gestacionais ${ }^{1}$.

O objetivo deste trabalho foi relatar um caso clínico ocorrido em quinta gestação de paciente, expondo a conduta da Equipe de Saúde de Família (ESF) para melhoria do conforto durante o período gestacional, bem como realização de revisão bibliográfica nas seguintes bases de dados: Lilacs, Medline, Biblioteca Cochrane e SciELO. Foram elegíveis artigos publicados no período de 2001 a 2011, com os seguintes indexadores: gigantomastia gestacional, macromastia gestacional, mastectomia bilateral simples, mamoplastia redutora e bromocriptina. Também foi efetuada a revisão manual em textos clássicos da especialidade. Em relação às considerações éticas, foram seguidos os princípios éticos, conforme Resolução 196/96 do Conselho Nacional de Saúde/ Ministério da Saúde (CNS/MS). Realizou-se a leitura do termo de consentimento livre e esclarecido, que foi obtido da paciente, para a publicação do relato de caso, garantindo-se a preservação dos dados, bem como o uso de figuras e imagens de forma que a paciente não pudesse ser identificada.

\section{Caso clínico}

Paciente parda, 29 anos, encaminhada para a ESF pelo serviço de mastologia do Hospital da Lagoa, no Rio de Janeiro (RJ), para acompanhamento pré-natal.
Iniciou pré-natal na ESF Parque Oswaldo Cruz (CMS Manguinhos) em 1 de março de 2011, queixando-se de aumento súbito do tamanho das mamas, dor e ferimentos. Paciente com 20 semanas gestacionais, Gesta V Para II A II, sendo dois partos vaginais e dois abortos no primeiro trimestre.

À consulta, portava exame ultrassonográfico transvaginal, realizado dia 1 de dezembro de 2010, apresentando feto único compatível com sete semanas de idade gestacional; ultrassonografia (USG) das mamas, realizada no Serviço de Mastologia do Hospital da Lagoa, de 28 de dezembro de 2010, com punção aspirativa de agulha fina (PAAF) em nódulo de mama direita e biópsia de mama direita com resultados inespecíficos, dermatite superficial perivascular linfocítica inespecífica e sem sinais de malignidade. A paciente foi tratada com cefalexina.

Ao exame físico, a gestante apresentava mamas de grande volume, pendulares; tecido epitelial hiperemiado difusamente e muito infiltrado (Figura 1); consistência endurecida, sugestiva de inflamação, afetando sua postura (Figura 2). Tumoração mal definida no quadrante superior lateral (QSL) da mama direita. Ausência de descarga papilar (Figura 3).

A gestante foi orientada pela equipe a manter as mamas elevadas, com curativo diário nas pequenas ulcerações e uso de analgésicos gerais quando necessário. A medida principal de cuidado é a contenção das mamas para o bemestar da paciente e acompanhamento do crescimento das mesmas, com visitas médicas a serem realizadas em intervalos curtos, em domicílio, com especial observação de sinais sugestivos de infecção.

A paciente foi encaminhada para o Hospital Maternidade Oswaldo Nazareth (Praça XV), onde iniciou pré-natal de alto risco em 14 de março de 2011.

Devido ao sofrimento materno, optou-se por agendar parto cesariano para 10 de junho de 2011, na $34^{a}$ semana de idade gestacional, tendo sido induzida maturação pulmonar fetal com corticosteroide. O resultado obstétrico foi feto vivo do sexo masculino, com boa vitalidade, pesando $2.190 \mathrm{~g}$ e comprimento de $44 \mathrm{~cm}$. O índice de Apgar tanto no primeiro quanto no quinto minuto foi de 9 . O recémnato permaneceu um dia na Unidade de Terapia Intensiva Neonatal, tendo alta com a mãe 72 horas após o parto.

A paciente recebeu cabergolina $1,0 \mathrm{mg}$, por dia (dois comprimidos de $0,5 \mathrm{mg}$, em dose única, administrados no primeiro dia após o parto), como prescrição para alta hospitalar. A paciente não iniciou a medicação por referir dificuldades financeiras e de aquisição. Face ao exposto pela paciente, o medicamento foi substituído pela ESF, devido ao alto custo e ao baixo poder aquisitivo da paciente, por bromocriptina em dose inicial de $2,5 \mathrm{mg} / \mathrm{dia}$ ( $1 / 2$ comprimido 
Figura 1. Mamas hiperemiadas, apresentando áreas de celulite e linfedema. Gravidez em 20ª semana de idade gestacional.

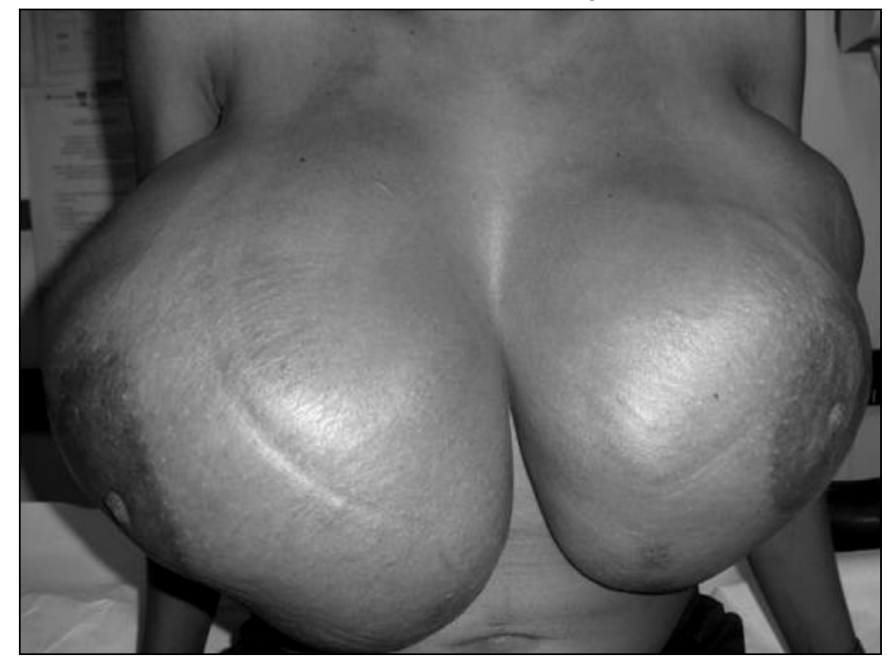

Figura 2. Visão lateral de mama esquerda.

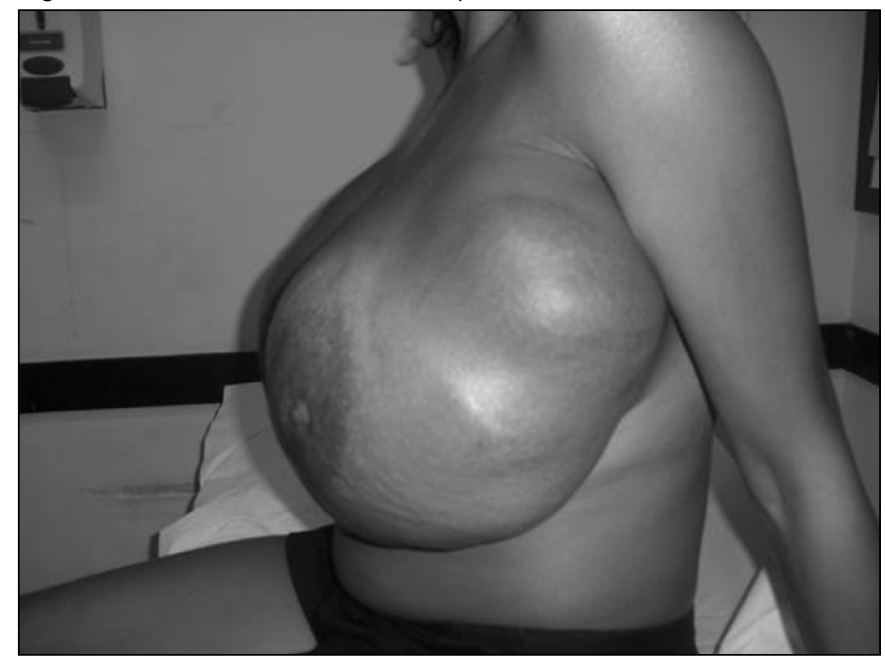

Figura 3. Visão lateral de mama direita. Tumoração mal definida em quadrante superior lateral.

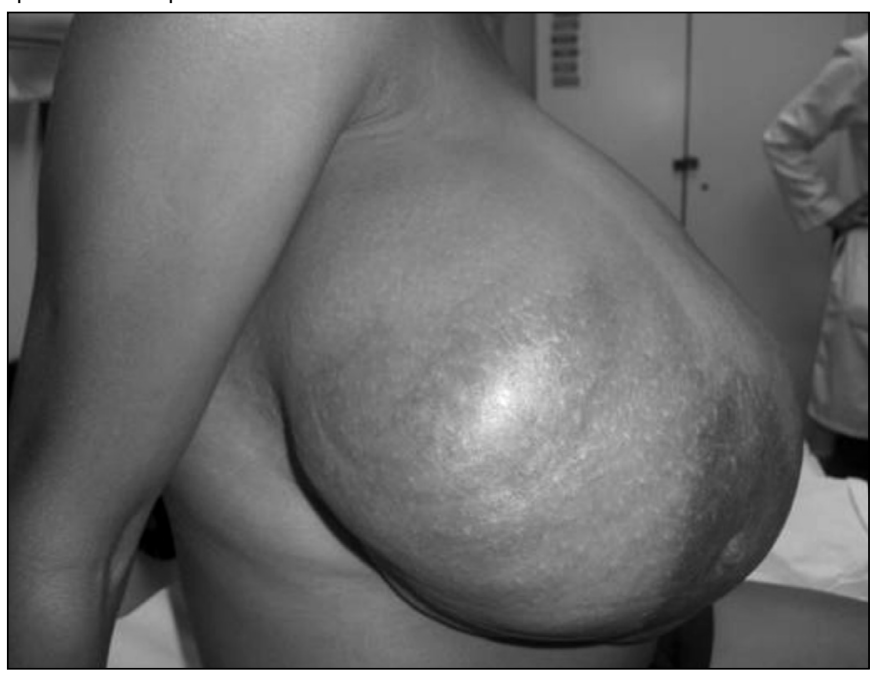

de 5,0 mg), aumentando-se gradativamente e alcançando a dose final de $5,0 \mathrm{mg}$, por dia, ao quarto dia de medicação. Após quatro dias de medicação prescrita pela ESF, a paciente apresentou melhora significativa da dor, parênquima mais macio à palpação e, embora hipertrofiadas, as mamas apresentaram volume nitidamente menor do que o observado durante a gestação. Ao longo do tratamento com bromocriptina, a paciente não referiu efeitos colaterais significativos, apenas leve desconforto gastrintestinal (cólicas) ao alcançar a dose final $(5,0 \mathrm{mg})$ no quarto dia de intervenção medicamentosa. Aguarda retorno ao Hospital da Lagoa, onde será submetida à mamoplastia redutora.

\section{Discussão}

A etiologia e a patogênese da gigantomastia gestacional são desconhecidas. A excessiva resposta dos receptores mamários aos hormônios produzidos inicialmente pelo corpo amarelo e, posteriormente, pela placenta parece ser o elemento desencadeador. Deste modo, diversos autores apresentam uma teoria hormonal, mesmo sem mecanismos exatos bem descritos ${ }^{6-8}$. Tanto os estrógenos quanto a gonadotrofina coriônica parecem estar envolvidos, mesmo com níveis séricos normais de acordo com a idade gestacional, o que também ocorre com a prolactina ${ }^{6}$.

Lewison et al. encontraram níveis de estrógenos elevados em tecido mamário de paciente com gigantomastia, bem como a contenção da hipertrofia com progestina, sugerindo uma hipersensibilidade dos receptores hormonais mamários $^{7}$. Lafreniere et $\mathrm{al}^{8}$. observaram níveis de receptores de estrógenos, progesterona e prolactina normais em amostras de biópsia de mamas com gigantomastia, embora os níveis séricos da prolactina estivessem elevados, ao passo que os estrógenos e a progesterona estavam normais. O crescimento das mamas foi contido com uso de bromocriptina, um inibidor de secreção de prolactina.

Outros autores propõem estímulos mediados por autoimunidade, pela relação com doenças autoimunes ou possível hipersensibilidade dos tecidos brancos à prolactina. A hipertrofia das mamas tem sido observada em mulheres não grávidas, tratadas com isoniazida, digitálicos e terapias com penicilamina para artrite reumatoide. Estas medicações parecem contribuir em maior estímulo estrogênico em glândulas mamárias e consequente crescimento das mamas. A etiologia é multifatorial com componente familiar. Casos associados ao lúpus eritematoso sistêmico, ao pseudo-hiperparatireoidismo e aos linfomas têm sido reportados. Deste modo, devem ser realizados: hemograma completo, provas de função hepática, eletrólitos e perfil hormonal ${ }^{9-11}$. 
O quadro clínico se caracteriza por aumento maciço, desproporcional em mamas, geralmente bilateral. A dor limita a atividade das pacientes, principalmente cefaleia, dor lombar e nos ombros. As pacientes apresentam transtornos de coluna dorsal e problemas posturais ${ }^{12}$. Pode-se ter aparecimento de úlceras, hemorragias, celulite, dor e necrose com risco de sepse, promovendo risco de vida tanto materna quanto fetal. A gigantomastia gestacional é preocupante para a paciente, a família e para os profissionais de saúde que assistem a família ${ }^{1}$.

$\mathrm{O}$ aspecto histopatológico do tecido mamário obtido por biópsia, mastectomia ou mamoplastia redutora é de hiperplasia glandular e importante aumento do tecido conectivo com ausência de tecido adiposo. Observam-se ductos e vasos linfáticos dilatados, fibroadenomatose e formações císticas ${ }^{4-6,60}$.

O tratamento proposto durante a gestação está orientado para melhorar as condições clínicas da paciente, em especial a dor e o auxílio psicológico. Além disso, deve-se estar atento às possíveis condições clínicas que aumentam o risco de morbimortalidade, as quais incluem anemia e sepse. Tem-se realizado ao longo dos anos o manejo farmacológico que inclui o uso de derivados de testosterona, estrógenos, tamoxifeno, hidrocortisona, diuréticos e supressores de prolactina. Entretanto, deve ser observada a efetividade questionável de algumas medicações, bem como o risco fetal ${ }^{1}$.

A bromocriptina é tida como uma droga de escolha. Tem-se observado parada de crescimento e, em alguns casos, regressão quando administrada diariamente após o parto. A bromocriptina em doses de 5,0 a $7,5 \mathrm{mg} /$ dia foi utilizada por diversos autores ${ }^{5,13,14}$.

No caso apresentado, a gestante iniciou acompanhamento pré-natal com intensa dor, que melhorou com as medidas já relatadas no caso clínico. As visitas médicas devem ser realizadas em intervalos curtos, com especial observação de sinais sugestivos de infecção. O acompanhamento com demais profissionais de ESF (nutricionista, psicólogo e enfermagem) é importante no manejo de possíveis complicações e na busca do resultado obstétrico desejável. Em casos de sinais de infecção, a antibioticoterapia deve ser rapidamente instituída.

A gestação deve ser levada a termo, aguardando o início espontâneo do trabalho de parto, ou induzi-lo em caso de sofrimento materno ou fetal. A mamoplastia re- dutora deve ser indicada, embora a involução espontânea tenha sido reportada ${ }^{5}$. Em caso de não involução, a intervenção cirúrgica na mama deve ser postergada após o quarto mês pós-parto ${ }^{12}$.

A atuação da equipe na ESF é muito importante para garantir conforto e apoio durante o período gestacional, melhorando as condições atuais da gestante, sobretudo a dor que a mesma provoca e o suporte psicológico, uma vez que, na vivência da mulher, a mama tem acentuada importância em sua imagem corporal e identidade feminina, o que pode abalar o sentimento de valor próprio, essencial para a saúde física e mental de uma mulher.

\section{Referências}

1. Vidaeff AC, Ross PJ, Livingston CK, Parks DH. Gigantomastia complicating mirror syndrome in pregnancy. Obstet Gynecol. 2003; 101(5 Pt 2): 1139-42.

2. Swelstad MR, Swelstad BB, Rao VK, Gutowski KA. Management of gestational gigantomastia. Plast Reconstr Surg. 2006; 118: 840-8. http:// dx.doi.org/10.1097/01.prs.0000232364.40958.47

3. Antevski BM, Smilevski DA, Stojovski MZ, Filipovski VA, Banev SG. Extreme gigantomastia in pregnancy: case report and review of literature. Arch Gynecol Obstet. 2007; 275(2): 149-53. http://dx.doi.org/10.1007/ s00404-006-0190-7

4. Zienert A. Macromastia in pregnancy: normal or a complication? Zentralbl Gynakol. 1990; 112(20): 1303-7.

5. Beischer NA, Hueston JH, Pepperell RJ. Massive hypertrophy of the breasts in pregnancy: report of 3 cases and review of the literature, "never think you have seen everything". Obstet Gynecol Surv. 1989; 44(4): 234-43. http:// dx.doi.org/10.1097/00006254-198904000-00002

6. Van der Meulen AJ. An unusual case of massive hypertrophy of the breasts. S Afr Med J. 1974; 48(34): 1465-6.

7. Lewison EF, Jones GS, Trimble FH, da Lima LC. Gigantomastia complicating pregnancy. Surg Gynecol Obstet. 1960; 110: 215-23.

8. Lafreniere R, Temple W, Ketcham A. Gestational macromastia. Am J Surg. 1984; 148(3): 413-8. http://dx.doi.org/10.1016/0002-9610(84)90484-7

9. Touraine P, Youssef N, Alyanakian MA, Lechat X, Balleyguier C, Duflos C et al. Breast inflammatory gigantomastia in a context of immune-mediated diseases. J Clin Endocrinol Metab. 2005; 90(9): 5287-94. http://dx.doi. org/10.1210/jc.2005-0642

10. Reyna Hinojosa R, Martínez Medellín J, Ceballos Quiroz R, Torres Huerta FR. Hipertrofia masiva de la glándula mamaria. Presentación de seis casos. Ginecol Obstet Mex. 1997; 65: 277-81.

11. Vandenberghe G, Claerhout F, Amant F. Lymphoblastic lymphoma presenting as bilateral gigantomastia in pregnancy. Int J Gynaecol Obstet. 2005; 91(3): 252-3. http://dx.doi.org/10.1016/j.jjgo.2005.07.017

12. Misirlioglu A, Akoz T. Familial severe gigantomastia and reduction with the free nipple graft vertical mammoplasty technique: report of two cases. Aesthetic Plast Surg. 2005; 29(3): 205-9. http://dx.doi.org/10.1007/ s00266-004-0134-1

13. Jido TA, Mohamed AZ, Alhasan SU. Gigantomastia complicating pregnancy: a case report. Niger J Med. 2006; 15(2): 167-9.

14. Ngarwal N, Kriplani A, Gupta A, Bhatla N. Management of gigantomastia complicating pregnancy. A case report. J Reprod Med. 2002; 47: 871-4. 